\title{
Corrigendum
}

\section{Consumption of fruit and berries is inversely associated with carotid atherosclerosis in elderly men}

I. Ellingsen, E. M. Hjerkinn, I. Seljeflot, H. Arnesen and S. Tonstad

doi: 10.1017/S0007114507832521 Published Online: 26 Sep 2007

In error, the following information was omitted from Tables 2 and 5 of this article.

In Table 2 the dietary and nutrient variables were $\ln$ transformed, all other variables were on the natural scale.

In Table 5 all variables were $\ln$ transformed. The value of B for total energy intake should be 0.054. 